\title{
Evidenz statt Eminenz
}

Medikamente müssen schon seit Jahrzehnten in klinischen Studien getestet werden. In der Chirurgie hinkt man ziemlich hinterher, weil jahrelang die Meinung einzelner Chirurgen wichtiger war als wissenschaftliche Transparenz. Doch gute Studien sind auch in der Chirurgie unerlässlich - man muss nur einiges beachten.

Felicitas Witte

Freie Medizinjournalistin

Korrespondenz: Dr. med. Felicitas Witte Pfeffingerstrasse 37 CH-4053 Basel Tel. 0793776193
Die Schweizer Gesellschaft für Chirurgie wird 100 Jahre alt. Es wird höchste Zeit, dass es auch in der Chirurgie bessere Studien gibt, fordern Chirurgen und Statistiker.

«Hören Sie auf mit dem neuen Zeug», sagt der Chirurg. «Das haben wir doch schon immer so gemacht - dabei bleibt es!» Niedergeschlagen schleicht der Oberarzt aus dem Zimmer seines Chefs. «Eminenzbasierte Chirurgie» statt evidenzbasiertes Handeln: Warum sich auf trockene Studiendaten verlassen, wenn man selbst Dutzende Male operiert hat und weiss, dass man perfekt ist? Eminenzbasierte Chirurgie stammt aus einer Zeit, in der Operateure noch nach eigenem Gusto vorgehen konnten und nicht von «bösen Statistikern» belehrt wurden.

Medikamente müssen schon seit Jahrzehnten in klinischen Studien getestet werden, bevor sie auf den Markt kommen. «Die Chirurgie hängt ziemlich hinterher», sagt Ralph A. Schmid, Präsident der Schweizerischen Gesellschaft für Chirurgie (SGC). Schon 2004 klagten Chirurgen im Deutschen Ärzteblatt, die Methodik der evidenzbasierten Medizin sei in der Chirurgie zu wenig umgesetzt [2]. Lange Zeit habe man sich auf Vorbilder wie Theodor Billroth und Theodor Kocher sowie verschiedene operative Schulen verlassen und diese als Meinungsbildner akzeptiert. Neue operative Verfahren werden oft nicht über einen transparenten und wissenschaftlich reproduzierbaren Weg in die Krankenversorgung übertragen. Das sollte sich ändern, und die Deutsche Gesellschaft für Chirurgie gründete im Jahre 2003 das Studienzentrum der Deutschen Gesellschaft für Chirurgie (SDGC).

«Es gab damals in Europa und den USA schon ähnliche Zentren», erzählt Markus Diener, Ärztlicher Leiter des SDGC. «Die waren aber meist interdisziplinär und vor allem onkologisch ausgerichtet.» So entschied man sich im SDGC, Studien zu operativen Strategien, chirurgischen Techniken oder Materialien durchzuführen - egal, ob es um Krebskrankheiten geht oder nicht. «Inzwischen haben Patienten und Kollegen erkannt, wie wichtig Studien in der Chirurgie sind», sagt Diener.

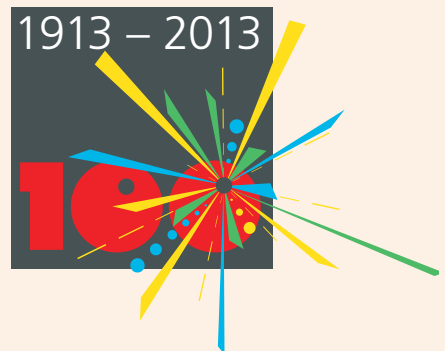

\section{Schweizerische Gesellschaft für Chirurgie (SGC/SSC)}

Die Schweizerische Gesellschaft für Chirurgie (SGC/SSC) hat in diesem Jahr ihren 100. Geburtstag gefeiert (www.sgc-ssc.ch). Zum Jubiläum erschien ein Sammelband über einhundert Jahre Chirurgie in der Schweiz mit medizinhistorischen Beiträgen, Reflexionen über die Chirurgie und Einschätzungen technischer Trends [1]. Die SÄZ veröffentlicht mit freundlicher Genehmigung des Chronos-Verlags Auszüge aus zwei Beiträgen. Wir beschliessen die kleine Reihe mit einem Text der Ärztin und freien Medizinjournalistin Felicitas Witte.

\section{Chirurgen lassen sich nicht so einfach vergleichen wie Tabletten}

Die beste Evidenz erzielt man - wie bei Medikamentenstudien - mit randomisierten kontrollierten Studien (RCT). «Studien in der Chirurgie sind aber nicht so einfach zu planen wie Medikamentenstudien», sagt Andreas Ziegler, Direktor des Instituts für Medizinische Biometrie und Statistik an der Uni Lübeck. Eines der grössten Probleme sei, dass man chirurgische Eingriffe nicht so gut standardisieren könne. «Vergleicht man zwei Medikamente, weiss man genau, wie viel in den Tabletten enthalten ist», sagt Ziegler. «Aber wie kann ich sicherstellen, dass Chirurg A genauso operiert wie Chirurg B?» Unterschiede gibt es nicht nur zwischen verschiedenen Spitälern, sondern auch innerhalb derselben Klinik. «Es ist schwierig festzulegen, ab wann ein Chirurg einen Eingriff so routiniert ausführt, dass er als Standard-Operateur〉 an einer Studie teilnehmen 


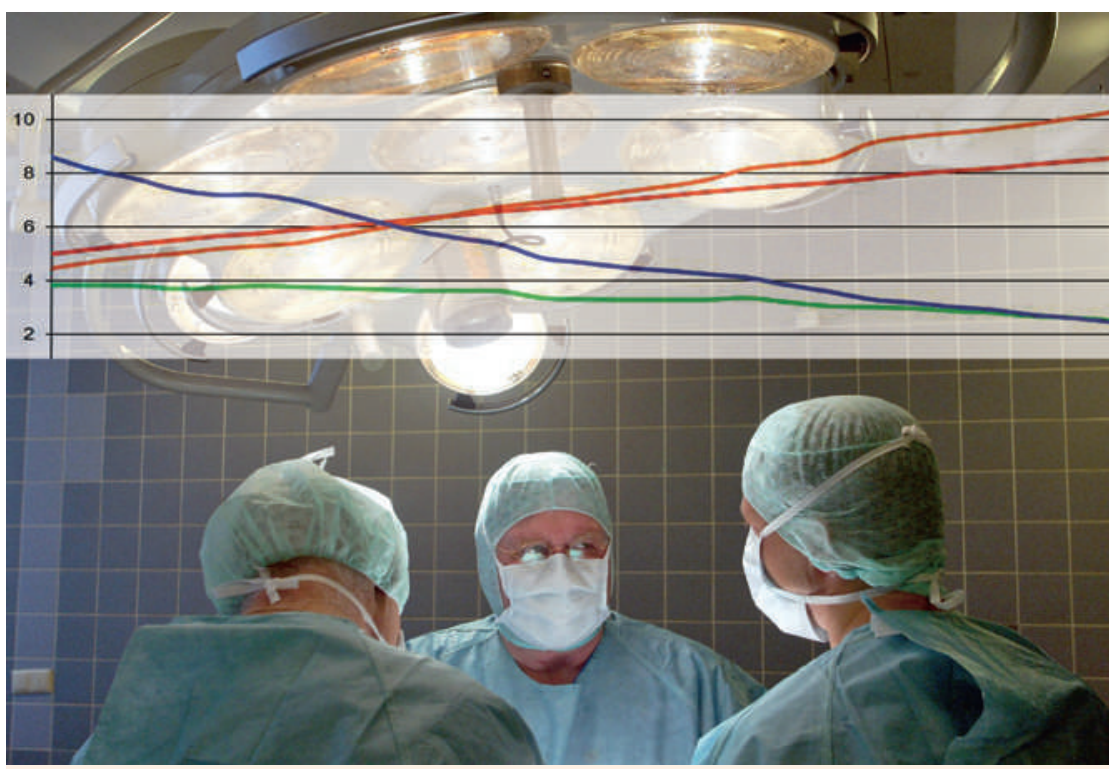

Ist die gewählte Operationsmethode wirklich die beste?

kann», sagt Ziegler. Hat ein Chirurg 30 Cholezystektomien durchgeführt, befähigt ihn das zwar, den Facharzt zu machen. Aber seine Operationstechnik lässt sich nicht ohne weiteres mit der seines Chefs vergleichen, der bereits mehr als 2000 Gallenblasen entfernt hat. Wie routiniert ein Chirurg ist, versuchen Statistiker bei der Randomisierung zu berücksichtigen. «Also beispielsweise, dass in beiden Gruppen ähnlich viele erfahrene und unerfahrene Chirurgen operieren», erklärt Ziegler. Besser sei es, wenn Chirurgen gegenseitig ihre Techniken anschauen und einen Standard entwickeln würden. Vorbildlich fand Ziegler das Vorgehen bei einer Studie in Japan, in der zwei verschiedene Techniken zur Gastrektomie verglichen werden sollten. «Die Japaner haben ihre Operateure in die Kliniken geschickt, um ihre Kollegen zu schulen», erzählt Ziegler. «Sie operierten langsam, damit alle sehen konnten, wie standardmässig operiert werden sollte.»

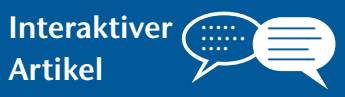

Wollen Sie diesen Artikel kommentieren? Nutzen Sie dafür die Kommentarfunktion in der OnlineVersion oder sehen Sie nach, was Ihre Kolleginnen und Kollegen bereits geschrieben haben: www.saez.ch/ aktuelle-ausgabe/ interaktive-beitraege/

\section{Die Chirurgen haben nicht genügend Zeit für Studientreffen.}

Hinzu kommt, dass ein chirurgischer Eingriff an sich komplexer ist als die Behandlung mit einem Medikament. «Man kann versuchen zu standardisieren», sagt Ziegler. «Aber man kann zum Beispiel nicht immer festlegen, welche Instrumente oder welches Nahtmaterial der Chirurg verwenden darf.»

Ein weiterer Aspekt ist die Verblindung. Man kann zwar einseitig verblinden und sicherstellen, dass der Patient nicht weiss, mit welcher Technik er operiert wurde. Doch der Chirurg ist sich natürlich immer bewusst, wie er operiert. «Zumindest muss man sicherstellen, dass der Nachuntersucher nicht weiss, welcher Patient mit welcher Technik operiert wurde», sagt Ziegler. Deshalb sei es auch so wichtig, die Endpunkte so objektiv wie möglich zu wählen: klar erfassbare Kriterien wie Todesfälle, Spital-Wiedereinweisungen, Entzündungen oder Blutungen.

\section{Neue OP-Methoden als Marketing-Instrument missbraucht}

Das grösste Hindernis, gute Studien durchzuführen, sieht der deutsche Statistiker jedoch im Arbeitsablauf der Chirurgen. «Um Studien zu planen, muss man sich in Ruhe an einen Tisch setzen», sagt Ziegler. Internisten hätten tagsüber immer mal zwischendurch Zeit. «Aber wenn ein Chirurg einen «Whipple» macht, ist vor abends nicht mit ihm zu rechnen.» So plant er Treffen mit den Chirurgen meist erst nach 18.00 Uhr am Abend. Doch oft klappt es selbst dann nicht. «Die Rahmenbedingungen sind an vielen Kliniken so schlecht, dass die Chirurgen nicht genügend Zeit für Studientreffen haben», klagt Ziegler. «Ohne den Input der Chirurgen geht es aber nicht.» Mal eben rasch ein Gespräch auf dem Gang über die geplante Studie, Telefonate mit dem Statistiker aus dem OP heraus, schnelle Gespräche mit den Studienkollegen zwischen zwei Patiententerminen unter solchen Rahmenbedingungen lässt sich keine Studie gut planen oder durchführen. Das SDGC in Deutschland zeigt, dass es funktionieren kann: «Hier ist immer ein Chirurg, der für Studiengespräche Zeit hat», sagt SDGC-Leiter Diener. «Für hochwertige klinische Studien in der Chirurgie müssen Chirurgen für die Forschung zumindest teilweise freigestellt werden.»

In der Schweiz fehle es an Zeit, Geld und Motivation, sagt SGC-Präsident Schmid. Gute Studien gäbe es, wenn eine Organisation wie die Schweizer Arbeitsgemeinschaft für Klinische Krebsforschung dahinter stehe oder ein ambitionierter Chirurg an einer Universität. An grösseren nichtuniversitären Kliniken lasse die Motivation aber zu wünschen übrig. «Viele wollen später Chefarzt in einem anderen Spital werden oder eine eigene Praxis eröffnen - da zählt Forschung nicht», sagt Schmid. Kleineren Spitälern gehe es primär um die Dienstleistung, «und viele Kliniken benutzen neue Operationsmethoden als Marketing-Instrument».

So bieten einige Chirurgen seit kurzem die «Single-Site-Cholezystektomie» an. Auf den Nabel setzt man ein Kunststoffgerät, durch das man Kamera und Instrumente gemeinsam einführt. Philippe Morel, ehemaliger Präsident der SGC, äusserte sich begeistert: Die Patienten hätten nicht nur weniger Narben, sondern man senke das Risiko von Blutungen, Narbenbrüchen und anderen Komplikationen. 
Ausserdem würden sich die Kranken schneller erholen. Das hatten Studien aber noch nicht belegt. Ernüchternd ist die systematische Übersicht von 13 RCTs von Chirurgen der Universität von Perugia [3]. Die Single-Site-Operation dauerte nicht nur länger, sie klappte auch öfter nicht, und die Patienten verloren mehr Blut. Keine der RCTs sei gut genug gewesen, um die Sicherheit des Eingriffs wirklich beurteilen zu können. Ausserdem ist die Single-Site-Operation teurer - nicht nur, weil sie länger dauert, sondern auch, weil man ein besonderes Zugangssystem braucht. Solange nicht belegt ist, dass die Patienten durch die neue Technik weniger Schmerzen und Komplikationen haben, sollte sie eigentlich nicht angewendet werden beziehungsweise nur in kontrollierten Studien. Es gibt viele solcher Beispiele.
Hat man erst einmal den Nutzen eines neuen chirurgischen Verfahrens gezeigt, kommt der nächste - oft schwierigere - Schritt, nämlich die Frage: Was darf das neue Verfahren kosten? «Die Diskussion, was wir uns leisten wollen oder können, muss man aus zwei Perspektiven führen», sagt SDGC-Leiter Diener. «Zum einen aus Patientensicht, zum anderen aus gesundheitspolitischer Sicht.» Die können mitunter völlig konträr sein. Manchmal würden auch die «Falschen» von neuen chirurgischen Verfahren profitieren, kritisiert Ralph Schmid. Durch die Einführung der laparoskopischen Chirurgie wurde die Hospitalisationszeit beispielsweise bei bestimmten Eingriffen von zehn Tagen auf ein bis drei Tage verkürzt. «Der «Gewinn» aus diesem Fortschritt bleibt aber nicht im Gesundheitswesen», sagt

\section{«Studien durchführen darf nicht mehr als zeitaufwendiges Hobby betrachtet werden.»}

\section{Von der randomisierten Studie zur Evidenz} Handeln ohne genügend Evidenz kann die Patientenversorgung gefährden und die Gesundheitskosten unnötig in die Höhe treiben, warnten kürzlich Chirurgen des Imperial College in London [4]. Statistische Unkenntnis, mangelhaftes Studiendesign, ethische Hürden, Probleme bei der Patientenrekrutierung, zu wenig Geld oder Zeitdruck sind nur einige der Hindernisse für gute RCTs. Hat man diese erst einmal überwunden, geht es um die zentrale Frage: Wie komme ich damit zur Evidenz? Denn auch eine noch so gute RCT bleibt eine einzelne Studie. Um die Evidenz aus RCTs zu bündeln, kennen Statistiker diverse Techniken, zum Beispiel Metaanalysen oder Netzwerk-Metaanalysen. Letztere erlauben es, zwei Behandlungsmethoden miteinander zu vergleichen, obwohl sie in den Einzelstudien nicht gegeneinander getestet wurden, sondern zum Beispiel nur gegen Placebo. Hat man Evidenz gewonnen, muss das natürlich auch im Alltag umgesetzt werden. Dazu gehört, dass Chirurgen über klinische Studien ausgebildet werden, dass sie genügend Zeit zum Forschen haben und wissen, wie sie die Ergebnisse interpretieren können. «Es gibt in der Schweiz eigentlich genügend Kurse für Chirurgen zur Weiterbildung in Statistik», sagt Ralph Schmid. «Aber das spricht nur wenige an.»
Schmid. «Zum einen profitiert die Industrie, die die Geräte herstellt, zum anderen die Volkswirtschaft, weil die Leute rascher wieder arbeiten können.» Je besser die Chirurgie werde, desto weniger werde die Leistung belohnt. Schmid fordert, der volkswirtschaftliche Gewinn müsse in neue Studien investiert und Chirurgen sollten für die Beteiligung an klinischen Studien bezahlt werden. «Studien durchführen darf nicht mehr als zeitaufwendiges Hobby betrachtet werden», sagt Schmid. «Dann würden bestimmt mehr Chirurgen forschen.»

\section{Literatur}

1 Steinke H, Schmid RA, Wolff E (Hrsg.). Schnitte, Knoten und Netze. 100 Jahre Schweizerische Gesellschaft für Chirurgie. Incisions, nœuds et réseaux. Les 100 ans de la Société Suisse de la Chirurgie. Zürich: Chronos; 2013. www.chronos-verlag.ch

2 Seiler C et al. Plädoyer für mehr evidenzbasierte Chirurgie. Dt. Ärzteblatt. 2004;101(6):A338-44.

3 Trastulli S, Cirocchi R, et al. Systematic review and meta-analysis of randomized clinical trials comparing single-incision versus conventional laparoscopic cholecystectomy. Br J Surg. 2013;100(2): 191-208.

4 Garas G et al. Evidence-based surgery: barriers, solutions, and the role of evidence synthesis. World J Surg. 2012;36(8):1723-31. 